INPLASY

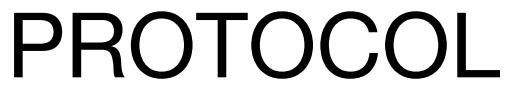

To cite: Zhang et al. Efficacy of Occlusal Splints in the Treatment of Temporomandibular

Disorders: a Systematic Review of Randomized Controlled Trials. Inplasy protocol 202040060 . doi: 10.37766/inplasy2020.4.0060

Received: 12 April 2020

Published: 12 April 2020

Corresponding author:

Kai-Xun He

kesonone@hotmail.com

Author Affiliation:

Fujian Medical University

Support: Fujian Province

Health System

Review Stage at time of this submission: Data analysis.

Conflicts of interest:

The authors report no conflict of interest.

\section{Efficacy of Occlusal Splints in the Treatment of Temporomandibular Disorders: a Systematic Review of Randomized Controlled Trials}

Zhang, SH¹; He, KX²; Lin, CJ33 Liu, XD; Wu, L5; Chen, J6; RauschFan, $\mathrm{XH}^{7}$.

Review question / Objective: P: patients with TMD as per RDC/TMD or DC/TMD criteria; I: occlusal splint; C: other treatment except occlusal splint; $O$ : pain, anxiety, movement. Condition being studied: Temporomandibular disorder (TMD) is a pathological condition involving the temporomandibular joint (TMJ), temporomandibular muscles, and nervous system. The prevalence of TMD across countries ranges from $10.5 \%$ to $54 \%$. The large variation of prevalence rates between countries may be due to different populations or examination methods, but more importantly, to different diagnostic criteria. Therefore, the International Network for Orofacial Pain and Related Disorders Methodology has recommended the uniform use of the Diagnostic Criteria for Temporomandibular Disorders (DC/TMD) and Research Diagnostic Criteria for Temporomandibular Disorders (RDC/TMD) in TMD clinical research and diagnosis. According to $\mathrm{DC} / \mathrm{TMD}$ and RDC/TMD, common TMD symptoms are TMJ noise, limitation of mandibular movement and pain, including arthralgia, myalgia, local myalgia, myofascial pain, myofascial pain with referral, headache attributed to TMD, which affect individual's quality of life and require treatment.

INPLASY registration number: This protocol was registered with the International Platform of Registered Systematic Review and Meta-Analysis Protocols (INPLASY) on 12 April 2020 and was last updated on 12 April 2020 (registration number INPLASY202040060).

\section{INTRODUCTION}

Review question / Objective: P: patients with TMD as per RDC/TMD or DC/TMD criteria; I: occlusal splint; C: other treatment except occlusal splint; $O$ : pain, anxiety, movement.
Condition be ing studied: Temporomandibular disorder (TMD) is a pathological condition involving the temporomandibular joint (TMJ), temporomandibular muscles, and nervous system. The prevalence of TMD across countries ranges from $10.5 \%$ to $54 \%$. The 
large variation of prevalence rates between countries may be due to different populations or examination methods, but more importantly, to different diagnostic criteria. Therefore, the International Network for Orofacial Pain and Related Disorders Methodology has recommended the uniform use of the Diagnostic Criteria for Temporomandibular Disorders (DC/ TMD) and Research Diagnostic Criteria for Temporomandibular Disorders (RDC/TMD) in TMD clinical research and diagnosis. According to DC/TMD and RDC/TMD, common TMD symptoms are TMJ noise, limitation of mandibular movement and pain, including arthralgia, myalgia, local myalgia, myofascial pain, myofascial pain with referral, headache attributed to TMD, which affect individual's quality of life and require treatment.

\section{METHODS}

Participant or population: Patients with TMD as per RDC/TMD or DC/TMD criteria.

Intervention: Occlusal splint.

Comparator: Other treatment except occlusal splint.

Study designs to be included: Randomized controlled trials.

Eligibility criteria: The eligibility criteria of studies were as follows: including subjects older than 18 years; diagnosed with TMD using well-established diagnostic criteria (DC/TMD or RDC/TMD); including an occlusal splint group and a proper control group; studies in English and were RCTs.

Information sources: Specific search terms and their combinations were used to search four databases: Medline via Pubmed, Web of Science, Embase and Scopus. Only included studies published after 1992. Keywords such as occlusal splint, temporomandibular joint disorders was used to search.

Main outcome(s): Pain, anxiety, movement of mandible.
Quality assessment / Risk of bias analysis: Joanna Briggs Institute's Critical Appraisal Checklist for Randomized Controlled Trials was used to assess the methodological quality of the included studies.

Strategy of data synthesis: Two authors collected the following data from the selected studies: publication year, location, occlusal splint design, mean age, sex, sample size, dropout rate, diagnostic criteria, TMD classification, groups, followup, outcomes, results, and conclusions.

Subgroup analysis: Occlusal splint group and other treatment group..

Sensibility analysis: None.

Country(ies) involved: China.

Keywords: Occlusal splints, movement disorders, joint pain, masticatory muscle disorders, temporomandibular joint disorders. 